\title{
HMM based scenario generation for an investment optimisation problem
}

\author{
Christina Erlwein • Gautam Mitra • Diana Roman
}

the date of receipt and acceptance should be inserted later

\begin{abstract}
The Geometric Brownian motion (GBM) is a standard method for modeling financial time series. An important criticism of this method is that the parameters of the GBM are assumed to be constants; due to this fact, GBM has been considered unable to properly capture important features, like extreme behaviour or volatility clustering. We propose an approach by which, the parameters of the GBM follow a regime switching model, more precisely a hidden Markov model (HMM). Thus, financial time series are modeled via a hidden Markov model (HMM) with a GBM in each state. Using this approach, we generate scenarios for a financial portfolio optimisation problem in which the portfolio CVaR is minimised. Numerical results are presented.
\end{abstract}

Keywords scenario generation, hidden Markov model, Geometric Brownian motion, asset allocation, optimal parameter estimation

\section{Introduction}

Stochastic programming is an important tool for portfolio optimisation. Since the future returns of most assets are uncertain, the outcome of an investment is uncertain. Usually, the decision maker tries to achieve an optimal combination of risk and expected return. This is done by solving stochastic programs: optimisation problems in which some of the parameters (the future returns) are not certain, but described by distributions. In order for the stochastic programs to be numerically solved, the distributions involved are approximated by discrete distributions with a finite number of outcomes (scenarios). An overview of financial optimisation problems can be found in (Mulvey (2001)).

Thus, a crucial issue for financial optimisation problems (with portfolio selection as a particular case) is the scenario generation for the future realisations of the time series involved. This is closely related to a proper modeling of the financial time series.

Among the most popular models for financial time series are the Geometric Brownian motion (GBM) (see for example Ross (2002)) and ARCH-GARCH models (Engle (1982), Bollerslev (1986)). With GBM, stock prices are approximated by continuous time stochastic processes. ARCH-GARCH models were designed for modelling time-dependent variance. However, both methods are accepted only with reservation; one of the main criticism is that they underestimate the lower tails of distributions, thus the severity of unfavourable

Christina Erlwein

Fraunhofer Institute, ITWM, Fraunhofer Platz 1, 67663 Kaiserslautern, Germany

Tel: +49 631 31600-4266 Fax: +49631316005352 E-mail: christina.erlwein@itwm.fraunhofer.de

Gautam Mitra · Diana Roman

CARISMA, Brunel University, Uxbridge, UB8 3PH, Great Britain and

OptiRisk Systems Ltd, Uxbridge, UB9 4DA, Great Britain

E-mail: gautam.mitra@brunel.ac.uk E-mail: diana.roman@brunel.ac.uk 
events. Both models may be used for scenario generation by sampling from the assumed distributions. A more recent method of scenario generation for general stochastic programs is the "moment-matching" approach (Hoyland et al (2003)) in which it is assumed that the moments of the "true" distribution are known; a discrete distribution with the same moments is then constructed. An overview of scenario generation for general stochastic programs is given in (Kaut and Stein (2003)).

Over the last years, hidden Markov models (HMM) became increasingly popular for modelling financial time series. One main idea behind these models is that some information on the market is hidden in noisy observations, e.g. price movements. The underlying hidden information can symbolise different stages of a business cycle, like expansion, peak, recession, trough and recovery, which influence the price movements. The market may switch from time to time from a state to another; each state is characterised by different parameters of the model for the observable stochastic process. In this case, one single model is valid only for short periods; regime switching models offer a better way of modelling.

A hidden Markov model (HMM) is a particular regime-switching model, in which there are two stochastic processes involved: apart from the one of interest, that is observable (e.g. asset prices), there is an underlying stochastic process describing the system's state over time, that is not observable, i.e. "hidden". HMMs were applied to a variety of fields, in particular to speech recognition (Rabiner (1989)); a review of HMMs and their applications is given in (Rabiner (2002)).

A review of the applications of HMM for financial time series modelling and forecasting is given in (Zhang (2004)). In (Hamilton and Susmel (1994)) weekly time series were modelled using regime switching with ARCH-type models within regimes. A simpler regime switching model for monthly returns with log-normal distributions is proposed in (Hardy (2001)). (Messina and Toscani (2007)) use autoregressive HMM for modeling univariate financial time series and for generating scenarios to describe their possible future evolution. In order to validate the HMM based scenario generation method, they show that the resulting Monte Carlo-sampled distributions can replicate with good approximation the empirical distribution of the observed data. In (Roman et al (2008)) multi-variate financial time series are modelled by using HMMs and mixtures of multi-variate normal distributions; based on this approach, scenarios are generated for a mean-downside risk optimisation problem. It is shown that the scenario generator based on this method has a reasonable stability and can capture extreme movements of asset prices. However, the resulting model is computationally demanding and can only handle a limited number of time series.

In this paper, we use HMM and Geometric Brownian motion for modelling financial time series. The motivation behind is that, although GBM is a traditional and popular method, it has recognised limitations. It fails to capture some important observed aspects, like volatility clustering and extreme behaviour. The limitations of GBM are mainly due to the fact that the parameters of the GBM (the "drift" and the volatility) are assumed to be constant. In our approach we aim to address this shortcoming by allowing the GBM parameters to switch between different states.

These parameters are estimated using a parameter estimation filtering approach (Elliott et al (1995)). Information of the Markov chain is filtered out of the observation process and optimal parameter estimates are derived with a reference probability measure technique. Once optimal parameters are estimated using historical information of the prices, scenario paths can be generated according to the parameter estimates and the filtered transition probability of the underlying Markov chain.

In our numerical experiments, we generate scenarios for the future returns of the FTSE100 index and of gold spots. The quality of the scenario generator is tested in a portfolio optimisation problem, in which the mean-CVaR approach is used, namely, the CVaR of the portfolio is minimised while a minimal portfolio expected return is imposed. We conclude that the scenario generation method proposed is reasonably stable.

The rest of the paper is organised as follows. In Section 2 the proposed approach for modelling financial time series is described. The basics of GBM and of HMM are presented. Once the parameter estimation and the future Markov chain estimation are made, the model can be used as a scenario generator. In Section 3 we describe the portfolio selection problem and the mean-CVaR optimisation model. Section 4 presents the numerical results. The scenarios generated with the approach presented in Section 2 are used as input in a mean-CVaR model; the stability of this scenario generator is investigated. Conclusions are presented in Section 5. 


\section{Modelling financial time series using HMM and GBM}

\subsection{The Geometric Brownian motion}

The Geometric Brownian motion (GBM) is a continuous time stochastic process that is widely used as a reasonable approximation of stock price dynamics. Although stock prices are stochastic processes in discrete time, this approximation is generally accepted by practitioners for short - medium time periods. Formally, a stochastic process $S_{t}$ follows a GBM if it satisfies the following stochastic differential equation:

$$
d S_{t}=\mu S_{t} d t+\sigma S_{t} d W_{t}
$$

where $W_{t}$ is a Wiener process, and $\mu$ (the "drift"), $\sigma$ (the volatility) are parameters of the model, assumed to be constant.

The equation (1) has the following analytic solution, where $S_{0}$ is the initial value (at time $t=0$ ):

$$
S_{t}=S_{0} \exp \left\{\left(\mu-\frac{\sigma^{2}}{2}\right) t+\sigma W_{t}\right\}
$$

One shortcoming of the model is that, in this form, it cannot account for observable phenomena in financial markets, like extreme behaviour and volatility clustering.

It is known that a Wiener process $W_{t}$ can be simulated as $\sqrt{t} z_{t}$, where $z_{t}$ is $\mathrm{N}(0,1)$ distributed. Thus, the GBM can be used for generating possible scenarios for $S_{t}$ by sampling from the standard normal distribution and using (2).

\subsection{Hidden Markov models with GBM as observation processes}

With the HMM approach, there are two stochastic processes involved (both in discrete time): in addition to the one of interest, which is observable (e.g. stock prices), there is another one describing the "state of the system", which is not directly observable. At each point in time, the system is in one of possible $n$ states and it may switch from the current state to another (or it may stay into the same state) according to some transition probabilities. Only an observation from the first stochastic process can be made. The underlying, hidden stochastic process describing the system's state is a Markov chain, i.e. the system's state at one point in time depends only on the system's state at the previous point in time and not on the entire history. Another assumption is that the transition probabilities are not time dependent (they only depend on the two involved states).

Each state generates observations for the stochastic process of interest according to its own probability density. In our approach, we assume that the observations of the stock prices are generated according to discretised Geometric Brownian motions; each state in the Markov chain has different parameters of the GBM. This approach may address the shortcomings of the GBM, described at the previous section.

We work on a complete probability space $(\Omega, \mathcal{F}, P)$. The log-return process $y_{k}=\ln \frac{S_{k}}{S_{k-1}}$ has the following dynamics in discrete time:

$$
y_{k+1}=f\left(\mathbf{x}_{k}\right)+\sigma\left(\mathbf{x}_{k}\right) z_{k+1} .
$$

where $f\left(\mathbf{x}_{k}\right)=\left(\mu\left(\mathbf{x}_{k}\right)-\frac{\sigma^{2}\left(\mathbf{x}_{k}\right)}{2}\right)$. The parameters $f$ and $\sigma$ are governed by the Markov chain $\mathbf{x}$ in discrete time and are therefore able to switch between different regimes. The $z_{k}^{\prime} s$ are a sequence of independent, identically distributed (IID) standard normal random variables independent of $\mathbf{x}$.

We denote the scalar product with $\langle\cdot, \cdot\rangle$, and with $n$ the number of states of the Markov chain. The parameters associated with GBMs are of the form $\mathbf{f}=\left(f_{1}, f_{2}, \ldots, f_{n}\right)^{\top}$ and $\boldsymbol{\sigma}=\left(\sigma_{1}, \sigma_{2}, \ldots, \sigma_{n}\right)^{\top}$ such that $f\left(\mathbf{x}_{k}\right)=\left\langle\mathbf{f}, \mathbf{x}_{k}\right\rangle$ and $\sigma\left(\mathbf{x}_{k}\right)=\left\langle\boldsymbol{\sigma}, \mathbf{x}_{k}\right\rangle$ where $\sigma_{i}$ 's are all positive for every $1 \leq i \leq n$. The observation at time $k+1$ depends on the state of $\mathbf{x}$ at time $k$. This is a one-step delay model and is reasonable as $y$ may not react to $\mathbf{x}$ immediately. 
We consider a homogeneous Markov chain $\mathbf{x}_{k}$ with finite state in discrete time, $k=0,1, \ldots$ The state space of $x_{k}$ is associated with the canonical basis $\left\{\mathbf{e}_{1}, \mathbf{e}_{2}, \ldots, \mathbf{e}_{n}\right\}$ of $\mathbb{R}^{n}$, where $\mathbf{e}_{i}=(0, \ldots, 0,1,0, \ldots, 0)^{\top} \in \mathbb{R}^{n}$ and $T$ denotes the transpose of a row vector, we suppose that the distribution of $\mathbf{x}_{0}$ is known. Let $\mathcal{F}_{k}^{0}=\sigma\left\{\mathbf{x}_{0}, \ldots, \mathbf{x}_{k}\right\}$ be the $\sigma$-field generated by $\mathbf{x}_{0}, \ldots, \mathbf{x}_{k}$, and $\mathcal{F}_{k}$ be the complete filtration generated by $\mathcal{F}_{k}^{0}$. Furthermore denote by $\mathcal{Y}_{k}$ the complete filtration generated by the sequence of increasing sub-sigma fields $\sigma\left(y_{1}, y_{2}, \ldots, y_{k}\right)$ from the observation process and let $\mathcal{G}_{k}=\mathcal{F}_{k} \vee \mathcal{Y}_{k}$ be the global filtration.

Under the real world probability measure $P$, the Markov chain $\mathbf{x}$ has the dynamics

$$
\mathbf{x}_{k+1}=\boldsymbol{\Pi} \mathbf{x}_{k}+\mathbf{v}_{k+1}
$$

where $\mathbf{v}_{k+1}:=\mathbf{x}_{k+1}-\boldsymbol{\Pi} \mathbf{x}_{k}$ is a martingale increment and $\Pi=\left(\pi_{j i}\right)_{j, i=1 \ldots n}$ is the transition probability matrix with $\pi_{j i}=P\left(\mathbf{x}_{k+1}=\mathbf{e}_{j} \mid \mathbf{x}_{k}=\mathbf{e}_{i}\right)$, i.e. the probability of transiting from state $j$ to state $i$ (as stated before, the assumption is that these transition probabilities are independent of time).

The parameters to be estimated are: the number of Markov states $n$, the transition probabilities $\pi_{j i}, j, i=1 \ldots n$ and the parameters of the discretised GBM's $\mathbf{f}$ and $\boldsymbol{\sigma}$. While optimal values can be derived recursively for f, $\boldsymbol{\sigma}$ and $\Pi$, the number of states is supposed to be determined in advance; they are estimated separately from the rest of the parameters. In practice, the state dimension of the hidden Markov chain may be dictated by the actual application or may be determined in an empirical manner by visual inspection of plots of the data (Messina and Toscani (2007), Geyer and Ziemba (2007)). In (Roman et al (2008)) the AIC criterion is used (Akaike (1974)) in order to determine the number of Markov states.

Recursive parameters can be derived for the remaining parameters $\mathbf{f}, \boldsymbol{\sigma}$ and $\Pi$, these parameter estimates use adaptive filters for the Markov chain. Filters are developed for the state space of the Markov chain and related quantities, e.g. the jump and the occupation time process. The jump process of the Markov chain, namely the number of times the Markov chain jumps from state $i$ to state $j$ up to a given time $k$ is denoted by $J_{k}^{(j i)}$.

$$
J_{k}^{(j i)}=\sum_{l=1}^{k}\left\langle\mathbf{x}_{l-1}, \mathbf{e}_{i}\right\rangle\left\langle\mathbf{x}_{l}, \mathbf{e}_{j}\right\rangle
$$

The occupation time process which indicates how long the Markov chain stays in state $i$ up to time $k$ is denoted by $O_{k}^{(i)}$.

$$
O_{k}^{(i)}=\sum_{l=1}^{k}\left\langle\mathbf{x}_{l-1}, \mathbf{e}_{i}\right\rangle
$$

Furthermore we need an auxiliary process $T_{k}^{(i)}$ for filtering the Markov chain.

$$
T_{k}^{(i)}(g)=\sum_{l=1}^{k}\left\langle\mathbf{x}_{l-1}, \mathbf{e}_{i}\right\rangle g\left(y_{l}\right)
$$

where $g$ is a function that will take the form $g(y)=y$ or $g(y)=y^{2}$.

Information for these processes is filtered out of our observation process $y$ under a reference probability measure $\widetilde{P}$ following an HMM filtering method by Elliott (Elliott et al (1995)). We define a new reference probability measure $\widetilde{P}$ such that the observation process is $\mathcal{N}(0,1)$ iid and independent from the Markov chain. Filter calculations are done under this reference probability measure, which is constructed by using a discrete-time version of Girsanov's theorem. A reverse measure change back to the real world measure $P$ then gives us the filter equation for the processes of the Markov chain under the physical measure, which are utilised for the parameter estimates. To construct the real world measure $P$ from $\widetilde{P}$, we define the processes $\lambda_{l}$ and $\Lambda_{l}$ by

$$
\lambda_{l}=\frac{\phi\left(\sigma\left(\mathbf{x}_{l-1}\right)^{-1}\left(y_{l}-f\left(\mathbf{x}_{l-1}\right)\right)\right)}{\sigma\left(\mathbf{x}_{l-1}\right) \phi\left(y_{l}\right)}
$$




$$
\Lambda_{k}=\prod_{l=1}^{k} \lambda_{l}, \quad k \geq 1, \quad \Lambda_{0}=1
$$

where $\phi(z)$ is the probability density function of the standard normal distribution. We refer to $\Lambda_{k}$ as the Radon-Nikodŷm derivative of $P$ with respect to $\widetilde{P},\left.\frac{d P}{d \widetilde{P}}\right|_{\mathcal{G}_{k}}=\Lambda_{k}$.

We shall work under the reference probability measure $\widetilde{P}$. For any $\mathcal{G}$-adapted process $\left\{H_{k}\right\}$ Bayes' Theorem gives us

$$
E\left[H_{k} \mid \mathcal{Y}_{k}\right]=\frac{\widetilde{E}\left[\Lambda_{k} H_{k} \mid \mathcal{Y}_{k}\right]}{\widetilde{E}\left[\Lambda_{k} \mid \mathcal{Y}_{k}\right]}
$$

so that the conditional expectation under the real world probability measure can be easily obtained.

Notation: We shall use the notation $\widehat{H}_{k}:=E\left[H \mid \mathcal{Y}_{k}\right]$ for any $\mathcal{Y}$-adapted process $H$ and $\gamma(H)_{k}:=$ $\widetilde{E}\left[\Lambda_{k} H_{k} \mid \mathcal{Y}_{k}\right]$.

Utilizing a filter theorem by Elliott (Elliott (1994)), we derive recursive filters for the jump process, the occupation time process and auxiliary processes. The filters $\gamma(H)_{k}$ are derived under the reference probability measure, therefore we use $\Lambda_{k}$, the Radon-Nikodŷm derivative of $P$, componentwise in our filter equations. For this, write $\mathbf{D}$ for the diagonal matrix whose $i$ th entry on the diagonal is

$$
\frac{\phi\left(\frac{y_{k+1}-f_{i}}{\sigma_{i}}\right)}{\sigma_{i} \phi\left(y_{k+1}\right)}
$$

First we would like to estimate the state of $\mathbf{x}$, given the observations under $P$, the real world probability. Let $\widehat{\mathbf{x}}^{i}=P\left(\mathbf{x}_{\mathbf{k}}=\mathbf{e}_{i} \mid \mathcal{Y}_{k}\right)=E\left[\left\langle\mathbf{x}_{\mathbf{k}}, \mathbf{e}_{i}\right\rangle \mid \mathcal{Y}_{k}\right]$ be the conditional distribution of $\mathbf{x}_{k}$ given $\mathcal{Y}_{k}$ under $P$. We define $\boldsymbol{\xi}_{k}:=\widetilde{E}\left[\Lambda_{k} \mathbf{x}_{k} \mid \mathcal{Y}_{k}\right]$. Noting that $\sum_{i=1}^{n}\left\langle\mathbf{x}_{k}, \mathbf{e}_{i}\right\rangle=1$, we have

$$
\sum_{i=1}^{n} \widetilde{E}\left[\left\langle\Lambda_{k} \mathbf{x}_{k}, \mathbf{e}_{i}\right\rangle\right]=\widetilde{E}\left[\Lambda_{k} \sum_{i=1}^{n}\left\langle\mathbf{x}_{k}, \mathbf{e}_{i}\right\rangle \mid \mathcal{Y}_{k}\right]=\widetilde{E}\left[\Lambda_{k} \mid \mathcal{Y}_{k}\right]=\sum_{i=1}^{n}\left\langle\boldsymbol{\xi}_{k}, \mathbf{e}_{i}\right\rangle
$$

Again noting that $\widehat{\mathbf{x}}_{k}=E\left[\mathbf{x}_{k} \mid \mathcal{Y}_{k}\right]$, we get an explicit form for the conditional distribution, $\widehat{\mathbf{x}}_{k}=\frac{\boldsymbol{\xi}_{k}}{\sum_{i=1}^{n}\left\langle\boldsymbol{\xi}_{k}, \mathbf{e}_{i}\right\rangle}$.

The filters for the processes are also derived by applying the change of measure technique. Filters are derived for $\gamma\left(H_{k} \mathbf{x}_{k}\right)_{k}$, but the estimate for $\gamma\left(H_{k}\right)$ is easily obtained by summing the components of $\gamma\left(H_{k} \mathbf{x}_{k}\right)_{k}$. With Elliott's filtering theorem (see Elliott (1994)), the following filters are derived:

$$
\begin{aligned}
\gamma\left(J^{(j i)} \mathbf{x}\right)_{l}= & \Pi \mathbf{D}\left(y_{l}\right) \gamma\left(J^{(j i)} \mathbf{x}\right)_{l-1} \\
& +\left\langle\boldsymbol{\xi}_{l-1}, \mathbf{e}_{i}\right\rangle \frac{\phi\left(\sigma_{i}^{-1}\left(y_{l}-f_{i}\right)\right)}{\sigma_{i} \phi\left(y_{l}\right)} \pi_{j i} \mathbf{e}_{j}, \\
\gamma\left(O^{(i)} \mathbf{x}\right)_{l}= & \Pi \mathbf{D}\left(y_{l}\right) \gamma\left(O^{(i)} \mathbf{x}\right)_{l-1} \\
& +\left\langle\boldsymbol{\xi}_{l-1}, \mathbf{e}_{i}\right\rangle \frac{\phi\left(\sigma_{i}^{-1}\left(y_{l}-f_{i}\right)\right)}{\sigma_{i} \phi\left(y_{l}\right)} \boldsymbol{\Pi} \mathbf{e}_{i} \\
\gamma\left(T^{(i)}(g) \mathbf{x}\right)_{l}= & \Pi \mathbf{D}\left(y_{l}\right) \gamma\left(T^{(i)}(g) \mathbf{x}\right)_{l-1} \\
& +\left\langle\boldsymbol{\xi}_{l-1}, \mathbf{e}_{i}\right\rangle \frac{\phi\left(\sigma_{i}^{-1}\left(y_{l}-f_{i}\right)\right)}{\sigma_{i} \phi\left(y_{l}\right)} g\left(y_{l}\right) \boldsymbol{\Pi} \mathbf{e}_{i} .
\end{aligned}
$$

with $\boldsymbol{\xi}_{k+1}=\boldsymbol{\Pi} \mathbf{D} \boldsymbol{\xi}_{k}$. More details and proofs for these filter derivations can be found in (Mamon et al (2008)).

The derived adaptive filters for the processes are used for the recursive model parameter estimates. The recursive formulas for the model parameters are calculated with a filter-based Expectation-Maximisation (EM)- algorithm. The maximum likelihood estimation (MLE) for the parameters within the EM-algorithm makes use of the derived adaptive filters of the observation process. In the calculation of the MLE, the 
filters substitute terms involving the observation process. The following recursive optimal parameter estimates for the transition probabilities $\pi_{j i}$, the mean $f_{i}$ and the variance $\sigma_{i}$ of the observation process are derived (see (Mamon et al (2008)) for a proof).

$$
\begin{gathered}
\widehat{\pi}_{j i}=\frac{\widehat{J}_{k}^{(j i)}}{\widehat{O}_{k}^{(i)}}=\frac{\gamma\left(J^{(j i)}\right)_{k}}{\gamma\left(O^{(i)}\right)_{k}} \\
\widehat{f}_{i}=\frac{\widehat{T}_{k}^{(i)}}{\widehat{O}_{k}^{(i)}}=\frac{\gamma\left(T^{(i)}(y)\right)_{k}}{\gamma\left(O^{(i)}\right)_{k}}
\end{gathered}
$$

and

$$
\widehat{\sigma}_{i}=\sqrt{\frac{\widehat{T}_{k}^{(i)}\left(y^{2}\right)-2 \widehat{f}_{i} \widehat{T}_{k}^{(i)}(y)+\widehat{f}_{i}^{2} \widehat{O}_{k}^{(i)}}{\widehat{O}_{k}^{(i)}}} .
$$

Parameters are re-estimated after a prespecified time interval. The algorithms run on these batches, new information available from the market is filtered and in turn utilized for the parameter estimate. When suitable initial values are chosen (according to statistical characteristics of the observed time series), the parameter estimates converge.

Once the parameters are estimated, this method can be used as a scenario generator for the future price returns, after the Markov chain for the next time period is estimated. As derived above, the conditional distribution of the Markov chain for the next time step is given by $E\left[\mathbf{x}_{k+1} \mid \mathcal{Y}_{k}\right]=\Pi \hat{\mathbf{x}_{k}}$ with $\hat{\mathbf{x}_{k}}=$ $E\left[\mathbf{x}_{k} \mid \mathcal{Y}_{k}\right]=\frac{\boldsymbol{\xi}_{k}}{\sum_{i=1}^{n}\left\langle\boldsymbol{\xi}_{k}, \mathbf{e}_{i}\right\rangle}$. Scenarios are then generated by sampling from the corresponding distributions, according to $(3)$.

\subsection{Algorithmic description}

The optimal parameter estimates are calculated through the following HMM algorithm:

1. Choose initial parameter values $\mathbf{f}_{0}, \sigma_{0}$ and $\Pi_{0}$. Here, the initial values are chosen with regard to the mean and standard deviation of the modelled time series, the initial transition probabilities are equally distributed.

2. Set initial values for the processes $J_{k}^{(j i)}, O_{k}^{(i)}$ and $T_{k}^{(i)}(g)$.

3. Choose length of batch (min $\sim 10$ data points) and number of algorithm runs depending on length of time series

4. Calculate the diagonal matrix $\mathbf{D}$ with entries from the Radon-Nikodŷm derivative $\Lambda_{k}$

5. Calculate the state estimator and filters for the jump-, the occupation time process as well as auxiliary processes (equations (13), (12) and (13).

6. After filters are calculated for the current batch, updates of the parameter estimates are obtained through equations (15), (16) and (14 utilising the adaptive filters. The resulting parameter estimates are taken as initial values in the next algorithm run.

7. Repeat the previous two steps on the next data batch, until the algorithm ran the chosen number of times.

8. The obtained optimal parameter estimates are utilised to generate scenarios for the returns of the financial time series.

The scenarios are generated through the following scenario generation algorithm:

1. Generate standard normal distributed random variables, which are used for simulating the white noise part (it is known that a Wiener process $W_{t}$ can be simulated as $\sqrt{t} \cdot N(0,1)$ )

2. Generate scenarios for the next time step:

a) the conditional distribution of the Markov chain for the next time step is calculated. As derived above, we have $E\left[\mathbf{x}_{k+1} \mid \mathcal{Y}_{k}\right]=\boldsymbol{\Pi} \hat{\mathbf{x}_{k}}$ with $\hat{\mathbf{x}_{k}}=E\left[\mathbf{x}_{k} \mid \mathcal{Y}_{k}\right]=\frac{\boldsymbol{\xi}_{k}}{\sum_{i=1}^{n}\left\langle\boldsymbol{\xi}_{k}, \mathbf{e}_{i}\right\rangle}$ 
b) the parameter values for the next time step are calculated as the scalar product between the conditional expectation of the Markov chain $\widehat{\mathbf{x}}_{k+1}$ and the estimated optimal parameters,

$$
f_{\text {scen }}=<f, \widehat{\mathbf{x}}_{k+1}>\text { and } \sigma_{\text {scen }}=<\sigma, \widehat{\mathbf{x}}_{k+1}>\text {. }
$$

c) the scenarios for the asset returns in the next time step are created with the discretised version of the geometric Brownian motion using the parameters stated above

$$
S_{\text {scen }}=C(k) * \exp \left(\left(f_{\text {scen }}-0.5 * \sigma_{\text {scen }}^{2}\right)+\sigma_{\text {scen }} * w_{\text {scen }}\right),
$$

where $C(k)$ denotes the last actual data point of the observation process and $w$ denotes the IID standard normal distributed random variable independent of the Markov chain for scenario scen.

d) the required log returns of the data series are then calculated for each scenario using the estimated prices calculated in the different scenarios above.

In our numerical experiments we consider two different frameworks for the scenario generations. In the first setting both time series are assumed to be independent, the optimal parameters for each process are derived in separate algorithms. The second setting assumes a dependency between the time series. Both observation processes are governed by the same Markov chain and are therefore estimated as vector observations. A thorough discussion on the filter derivation and optimal parameter estimation for vector observations can be found in (Elliott et al (1995)) and (Erlwein and Davison (2009)).

\section{The mean-CVaR model for portfolio optimisation}

A crucial issue in financial optimisation is the choice criterion used. The result of a financial decision (say a portfolio return) is uncertain and regarded as a random variable (with a discrete distribution, using scenario generation). Thus a natural question is what criterion to use for making choices among random returns. Various models for optimal portfolio selection have been proposed, starting with the popular Mean-Variance approach (Markowitz (1952)), in which decisions (i.e., portfolio weights) are taken such that the portfolio's expected return is maximised while a risk measure (the variance) is minimised. More recently portfolio optimisation problems include more sophisticated risk measures. A popular risk measure in industry at the moment is Value-at-Risk ( VaR), which indicates the maximum amount to be lost at a particular confidence level. One drawback of this measure in the application to stochastic optimisation problems is that it is non-smooth and non-convex and can therefore lead to multiple local extrema (Artzner et al (1999)). On the other hand, the Conditional Value-at-Risk (CVaR), also known as Mean Excess Loss or Mean shortfall, which is approximately equal to the conditional expectation of losses beyond VaR, is a risk measure with good theoretical and computational properties (Pflug (2000)). CVaR models were developed for portfolio optimisation, where in one framework (Rockafellar and Uryasev (2000)) risk is minimised with a given minimum level of portfolio return, while in other frameworks (Krokhmal et al (2002)) the return is maximised with a given maximum level of risk, or a weighted combination of risk and return is optimised. All formulations lead to the same efficient frontier (Krokhmal et al (2002)).

Let $R_{x}$ be a random variable representing the return of a portfolio $x$ over a given holding period and $A \%=\alpha \in(0,1)$ a percentage which represents a sample of "worst cases" for the outcomes of $R_{x}$ (usually, $\alpha=0.01=1 \%$ or $\alpha=0.05=5 \%)$.

The definition of CVaR at the specified level $\alpha$ is the mathematical transcription of the concept "average of losses in the worst $A \%$ of cases" (Acerbi and Tasche (2002)), where the "loss" associated with $R_{x}$ is usually described by the random variable $-R_{x}$, meaning a negative return is a loss, a positive return is a "gain" (however, the definition of CVaR allows for a general loss function.)

For a detailed definition of CVaR, see (Rockafellar and Uryasev (2002)). CVaR is approximately equal to the average of losses greater than or equal to $\mathrm{VaR}$ (at the same confidence level $\alpha$ ); in some cases, the equality is exact.

An important result, proved in (Rockafellar and Uryasev (2002)), is that the CVaR of a portfolio $x$ can be calculated by solving a convex optimisation problem. Moreover and very importantly, CVaR can be 
optimised over the set of feasible decision vectors (feasible portfolios) and this is also a convex optimisation problem. In (Rockafellar and Uryasev (2002)), an auxiliary function is used, $F: X \times \mathbb{R} \rightarrow \mathbb{R}$,

$$
F_{\alpha}(x, v)=\frac{1}{\alpha} E\left[-R_{x}+v\right]^{+}-v
$$

where $[u]^{+}=u$ if $u \geq 0$ and 0 otherwise. They proved that minimising CVaR over $X$ can be done by minimising $F_{\alpha}$ over $X \times \mathbb{R}$.

When the random returns under consideration are represented as discrete random variables (via scenario generation), the CVaR optimisation problem is a LP.

The algebraic formulation of the mean-CVaR model is given below:

$$
\begin{gathered}
\min \quad v+\frac{1}{\alpha S} \sum_{i=1}^{S} y_{i} \\
\text { Subject to: } \\
\sum_{j=1}^{N}-r_{i j} x_{j}-v \leq y_{i}, \quad \forall i \in\{1 \ldots S\} \\
y_{i} \geq 0, \quad \forall i \in\{1 \ldots S\} \\
\sum_{j=1}^{N} \mu_{j} x_{j} \geq d ; \quad x \in X .
\end{gathered}
$$

where the parameters of the model are:

- $S=$ the number of scenarios

- $N=$ the number of assets

- $r_{i j}=$ return of asset $j$ under scenario $i, i=1 \ldots S, j=1 \ldots N$

- $\mu_{j}=$ the expected return of asset $\left.j, j=1 \ldots N\left(\mu_{j}=\frac{1}{S} \sum_{i=1}^{S} r_{i j}\right)\right)$

- $d=$ the desired expected return of the portfolio

The decision variables of the model are:

- $x_{j}=$ the fraction of portfolio wealth invested in asset $j, j=1 \ldots N$

- $v=$ the negative of an $\alpha$-quantile of the portfolio return distribution (it is an approximation for the $\alpha$-VaR of the portfolio)

- $y_{i}=$ the magnitude of the negative deviations of the portfolio return from the $\alpha$-quantile, for every scenario $i \in\{1 \ldots S\}$ (they are 0 if the portfolio return is higher than the $\alpha$-quantile)

\section{Numerical experiments: A case study for an investment problem}

We consider the following portfolio optimisation problem. An investor faces the problem of finding the optimal ratio between investing in gold spots, FTSE100 stocks or putting his money into a bank account. We consider a one time-step problem: decisions have to be taken now in order to give the best return at the next time period.

An investment in gold is traditionally seen as a long-term investment that can play a role in hedging against inflation and political or economical problems. Like other commodities, gold is traded on spot and future markets, but it has specific characteristics which are not common within commodity markets. Because of its role as a global currency, prices on spot markets are global. Gold supply and demand does not depend on seasonality, it has a low risk of supply interruption and low storage and insurance costs. Furthermore gold has no risk of spoilage and the consumption level relative to inventory is low. Unlike price processes for other commodities, a convenience yield is not included in the price process due to the distinctive gold 
features.

We generate scenarios for the future prices of gold and FTSE100 index according to the scenario generation method proposed in section 2. These scenarios are further used in a mean-CVaR optimisation model (section 3) in order to take the required decisions.

The dataset used for generating the scenarios are historical prices of gold spots and FTSE 100 index, monitored daily over a 1-year period. We considered a number of $n=3$ possible states for the Markov chain. This is consistent with Roman et al (2008), Messina and Toscani (2007), Geyer and Ziemba (2007), who, either by inspecting data or by using statistical techniques, estimated the number of Markov states to 3. It is in fact more or less agreed that three states usually gives a good representation of asset prices, without overfitting the model.

We generate scenarios under two different frameworks. First, we consider that the two time series of interest are independent and thus governed by different Markov chains. Secondly, we consider them governed by the same Markov chain; observations are generated as vectors.

The algorithm for estimating the parameters runs 24 times on the data sets. For each data set the parameters are updated when new information arrives after batches of 10 data points. Scenarios are generated starting from the last data point. Figures 1 and 2 depict the optimal parameter estimates for the gold spot prices and for FTSE 100, respectively (estimated independently). Scenarios for the next time period are generated following the parameter estimation.

We generate 10,000 paths for both time series, the gold spot prices and FTSE 100. For this we simu-
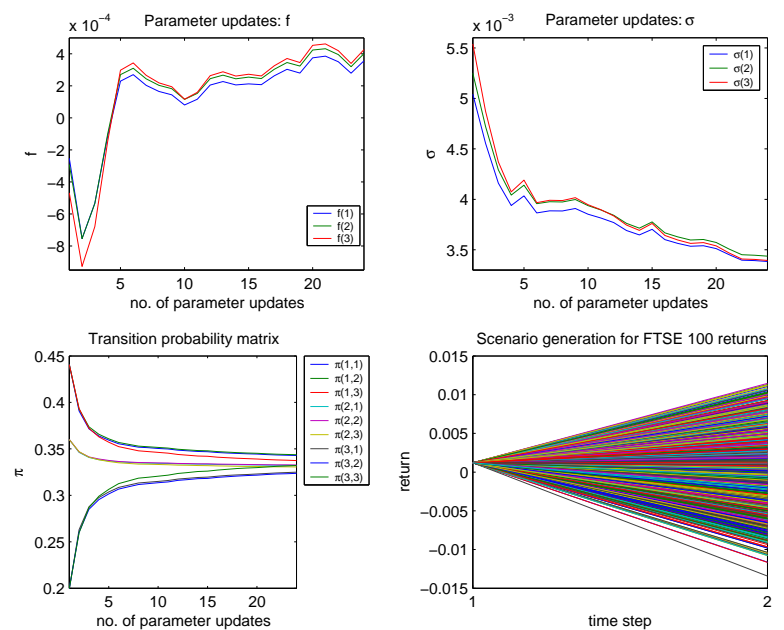

Fig. 1 Parameter estimation and scenario generation for FTSE 100.
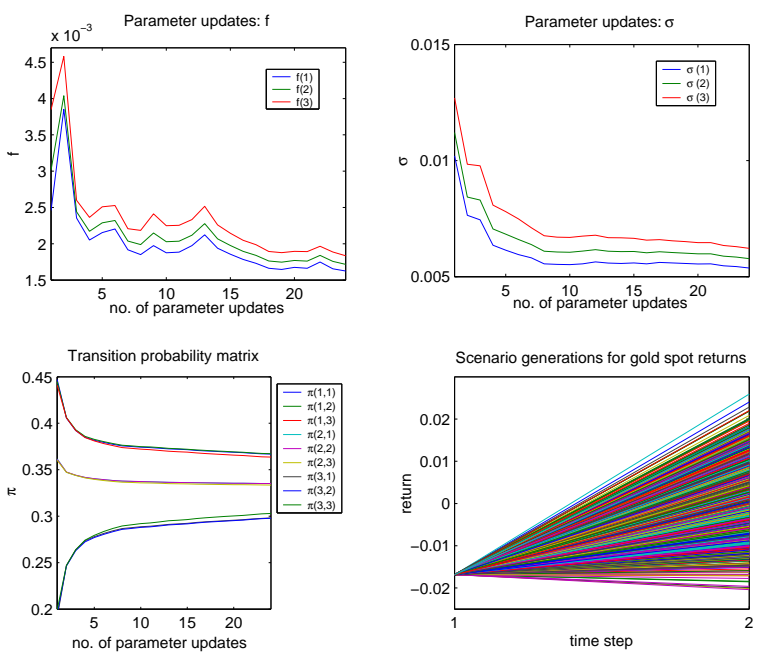

Fig. 2 Parameter estimation and scenario generation for gold spots 
late a 3-state Markov chain which follows the estimated transition probabilities and with this we simulate paths of the asset prices which take into account the optimal parameter estimates of the time series. A comparison of the descriptive statistics of these paths and the actual time series can be found in table 1 , ten sample paths for each time series are plotted in figure 3.

\begin{tabular}{r|r|r|r|r|r|r|r}
\hline & Min & Max & Median & Mean & St.dev & Skewness & Kurtosis \\
\hline \hline Gold spot & & & & & & & \\
Actual time series & 524.75 & 725.00 & 609.60 & 603.77 & 39.95 & 0.2123 & 2.5406 \\
Mean of generated paths (3-state) & 488.89 & 762.82 & 609.44 & 613.77 & 70.96 & 0.1681 & 2.3906 \\
Mean of generated paths (1-state) & 526.77 & 859.71 & 672.24 & 678.83 & 94.79 & 0.1701 & 1.9651 \\
\hline \hline FTSE & & & & & & & \\
Actual time series & 5506.8 & 6260.0 & 5896.8 & 5920.3 & 180.13 & 0.0443 & 2.1054 \\
Mean of generated paths (3-state) & 5281.9 & 6376.1 & 5805.0 & 5811.6 & 273.47 & 0.0502 & 2.4129 \\
Mean of generated paths (1-state) & 5637.2 & 6676.8 & 6136.0 & 6140.9 & 288.55 & 0.0607 & 2.1337 \\
\hline
\end{tabular}

Table 1 Descriptive statistics of generated paths for gold spot prices and FTSE
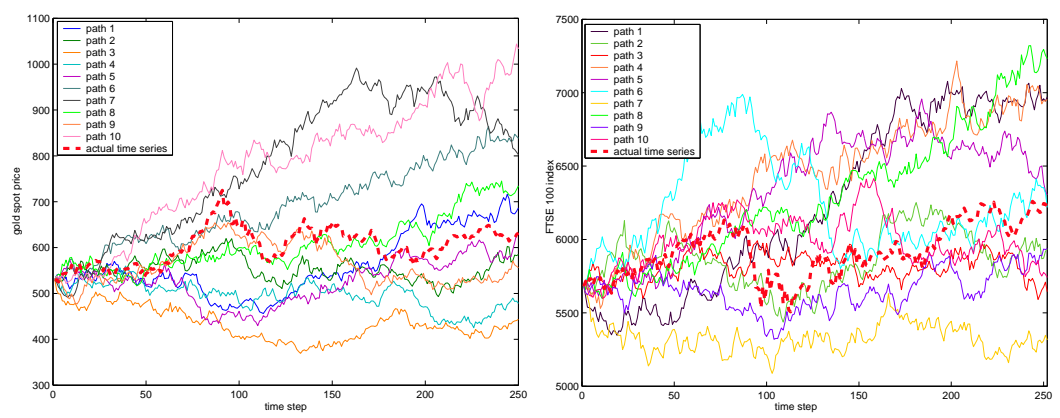

Fig. 3 Sample paths for gold spots and FTSE 100 index within 3-state framework.

Under the assumption of correlated time series, we consider that both time series are governed by the same hidden Markov chain, which is filtered out from both processes. Under this framework we estimate the optimal parameters of both observation processes simultaneously and we generate vector observations. Figure 4 shows the parameter estimation of the observation processes for the vector observation case. Possible scenarios for both time series are then generated and depicted in figure 5.

4.1 Stability of the scenario generators

Stability is one of the most important requirements from a scenario generator. Since any scenario generator has an element of randomness, the final outcome depends on the values drawn from the corresponding distributions (in our case, the standard normal distribution). Thus, every time a scenario generator is run, different sets of scenarios are obtained. Stability guarantees that the optimal solution of the optimisation problem of interest does not depend (but, possibly, only to a small extent) on the specific scenario set chosen.

For each framework (independent / correlated time series) we use the same methodology. We generate 10 sets, each with 5000 scenarios for the future returns of FTSE100 and gold. The scenarios obtained are used as input in the mean-CVaR optimisation problem (the scenarios are considered to have equal probability). We consider CVaR at confidence level $\alpha=1 \%$. The daily return on the bank account is assumed to be 

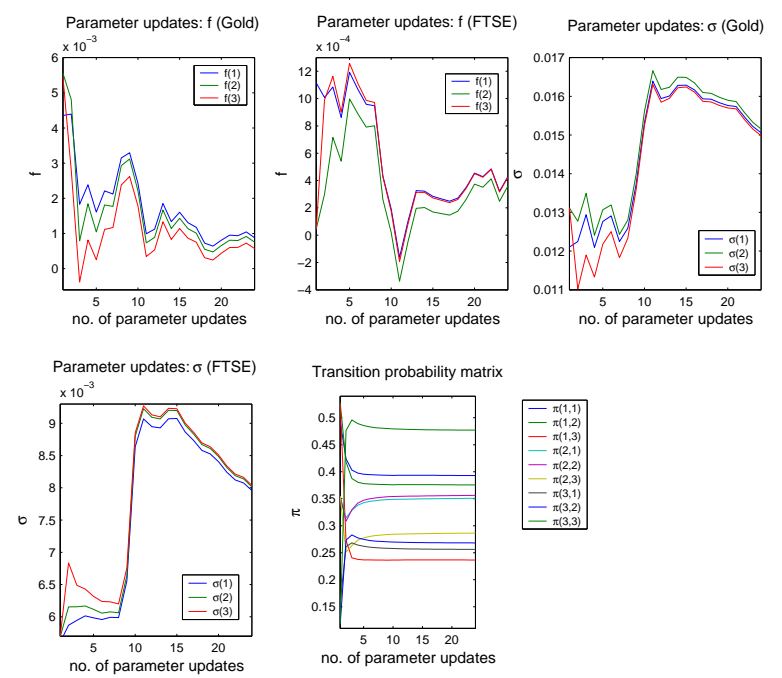

Fig. 4 Simultaneous parameter estimation for observation process of gold spots and FTSE 100 returns
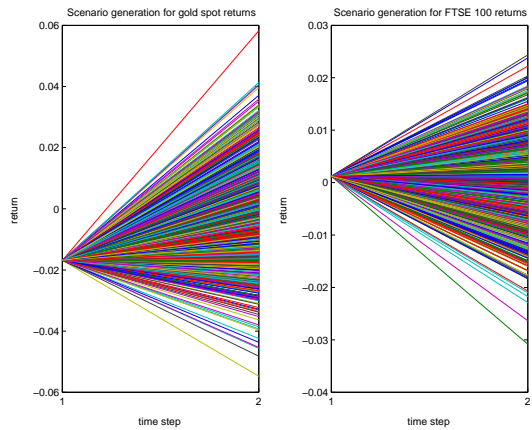

Fig. 5 Scenario generation for returns on gold spots and FTSE 100

0.0001. The minimal required level of daily portfolio return is set to $d=0.0005$. The optimisation problems are formulated in AMPL (Fourer et al (1989)) and solved with the FortMP solver (Ellison et al (1999)).

The optimal solutions and objectives, for the case of independent observations, are displayed in Table 2. All values of the objective function lie between 0.002844 and 0.003325 with a mean of 0.0031 and variance of $2.7070 e-008$. The in-sample stability of the scenario generator is further investigated through a variation of the size of the scenario sets. The mean, standard deviation, minimum and maximum of the resulting objective functions are depicted below in Table 3. The value of the objective function is determined for ten different scenario sets of each length.

The optimal solutions and objectives, for the case of vector observations, are displayed in Table 4. All values of the objective function lie between 0.015058 and 0.027259 with a mean of 0.0193 and variance of $1.4093 e-005$. The in-sample stability of the scenarios generated through the vector setting is further examined through varying sizes of scenario sets. The descriptive statistics of the resulting objective functions are depicted in table 5 .

It is evident from the tables above that the scenario generator is more stable under the framework of independent observations. The stability under this framework is good, with respect to both the optimal percentages to be invested and the optimum. The similarity of optimal solutions guarantees a good outof-sample stability of this scenario generator (i.e. stability with respect the "true", unknown distribution of the random returns), which is one of the most important aspects. The examination of scenario sets of different sizes shows a good in-sample stability of both settings, however, the independent settings leads to lower standard deviations and a smaller range.

When generating scenarios through vector observations, there is a higher variability of both optima and optimal solutions. Economically this can be explained by the largely uncorrelated price of gold to any 


\begin{tabular}{|c|c|c|c|c|c|c|c|c|c|c|}
\hline Scenario set & 1 & 2 & 3 & 4 & 5 & 6 & 7 & 8 & 9 & 10 \\
\hline Objective func. & 0.00333 & 0.00321 & 0.00296 & 0.00320 & 0.00322 & 0.00304 & 0.00300 & 0.00284 & 0.00293 & 0.00290 \\
\hline $\begin{array}{l}\text { Investment in } \\
\text { FTSE (in \%) }\end{array}$ & 9.77 & 17.64 & 9.14 & 16.88 & 15.27 & 12.03 & 15.84 & 10.38 & 13.63 & 8.81 \\
\hline Gold (in \%) & 24.37 & 20.71 & 22.26 & 21.80 & 22.76 & 23.08 & 21.08 & 21.44 & 21.80 & 22.38 \\
\hline Bond (in \%) & 65.96 & 61.65 & 68.60 & 61.32 & 61.97 & 64.89 & 63.08 & 68.18 & 64.57 & 68.81 \\
\hline
\end{tabular}

Table 2 Stability analysis for 10 scenario sets with independent observations

\begin{tabular}{l|l|l|l|l}
\hline Objective function: & Mean & Std dev & Min & Max \\
\hline 500 scen & 0.00323 & 0.00083 & 0.00240 & 0.00491 \\
1000 scen & 0.00312 & 0.00044 & 0.00263 & 0.00387 \\
2000 scen & 0.00307 & 0.00029 & 0.00272 & 0.00352 \\
5000 scen & 0.00306 & 0.00016 & 0.00284 & 0.00332 \\
\hline
\end{tabular}

Table 3 In-sample stability of scenario sets in independent setting

\begin{tabular}{|c|c|c|c|c|c|c|c|c|c|c|}
\hline Scenario set & 1 & 2 & 3 & 4 & 5 & 6 & 7 & 8 & 9 & 10 \\
\hline Objective func. & 0.01993 & 0.02154 & 0.02154 & 0.01357 & 0.01793 & 0.02405 & 0.02148 & 0.02024 & 0.018912 & 0.02154 \\
\hline $\begin{array}{l}\text { Investment in } \\
\text { FTSE (in \%) }\end{array}$ & 21.66 & 35.36 & 21.66 & 47.54 & 41.43 & 33.15 & 21.38 & 56.16 & 48.25 & 21.66 \\
\hline Gold (in \%) & 52.34 & 45.34 & 52.34 & 23.14 & 41.40 & 62.41 & 55.62 & 42.63 & 41.83 & 52.34 \\
\hline Bond (in \%) & 26.00 & 19.30 & 26.00 & 29.32 & 17.17 & 4.44 & 23.00 & 1.22 & 9.93 & 26.00 \\
\hline
\end{tabular}

Table 4 Stability analysis for 10 scenario sets with vector observations

\begin{tabular}{l|l|l|l|l}
\hline & Mean & Std dev & Min & Max \\
\hline 500 scen & 0.0191 & 0.0090 & 0.0078 & 0.0357 \\
1000 scen & 0.0190 & 0.0061 & 0.0109 & 0.0319 \\
2000 scen & 0.0188 & 0.0045 & 0.0142 & 0.0261 \\
5000 scen & 0.0201 & 0.0028 & 0.0136 & 0.0241 \\
\hline
\end{tabular}

Table 5 In-sample stability of scenario sets in vector setting

other stock. Due to its characteristics as a stable long-term investment and a global currency, gold prices have a low correlation to stock prices or indices. Therefore optimal solutions calculated with independent generated scenarios are more stable.

We extended our numerical study to two correlated indices, namely FTSE 100 and Eurostoxx 50. It has to be noted that also in that case where highly correlated indices are used in these scenario generation methods, the objective function reaches a smaller minimum with independently generated scenarios, the values of the objective functions are in smaller ranges. Nevertheless the distribution of generated paths for correlated indices are closer to the actual distributions observed in the market. Table 6 depicts the descriptive statistics of sets of 1,000 sample paths. Here we distinguish between sample paths generated from the hidden Markov model with vector observations (labelled '2-dim') and sample paths generated independently for FTSE 100 and Eurostoxx 50. The characteristics of actual data is closely reached through paths generated with estimated parameters from the vector setting.

\begin{tabular}{r|r|r|r|r|r|r|c}
\hline & Min & Max & Median & Mean & St.dev & Skewness & Kurtosis \\
\hline \hline Eurotoxx & & & & & & & \\
Actual time series & 3408.0 & 4140.7 & 3792.0 & 3795.6 & 173.56 & 0.1351 & 2.2448 \\
Mean of generated paths (2-dim) & 3348.7 & 4274.3 & 3779.2 & 3787.7 & 235.56 & 0.1115 & 2.3728 \\
Mean of generated paths (1-dim) & 3591.0 & 4737.5 & 4119.4 & 4135.0 & 329.98 & 0.1074 & 1.9170 \\
\hline \hline FTSE & & & & & & & \\
Actual time series & 5506.8 & 6260.0 & 5896.8 & 5920.3 & 180.13 & 0.0443 & 2.1054 \\
Mean of generated paths (2-dim) & 5335.8 & 6572.0 & 5924.4 & 5930.2 & 315.13 & 0.0587 & 2.3782 \\
Mean of generated paths (1-dim) & 5281.9 & 6376.1 & 5805.0 & 5811.6 & 273.47 & 0.0502 & 2.4129 \\
\hline
\end{tabular}

Table 6 Descriptive statistics of generated paths in a one- and two-dimensional setting for Eurostoxx 50 and FTSE 100 
In this section we modify our original investment problem and add constraints on the weights (fractions of capital) invested in the different assets. All weights now have an upper bound of $50 \%$, i.e. no more than half of the budget is allowed to be invested in either gold spots, FTSE 100 stocks or bonds. Taking into account the results from the previous section, we only use the scenario generator that assumes independent observations. We generate five sets containing 5,000 scenarios and five sets containing 20,000 scenarios; we solve the constrained mean-CVaR optimisation problem with this input. As it may be seen from Table 7, the stability of the solutions and objective is very good. The average objective function over all generated scenario sets is 0.003538409 with a very low variance of $3.77072 e-09$. As already specified in the previous section, the similarity of the optimal solutions implies a good out-of-sample stability.

\begin{tabular}{|c|c|c|c|c|c|c|c|c|c|c|}
\hline \multirow[b]{2}{*}{ Scenario set } & \multicolumn{5}{|c|}{5,000 generated scenarios } & \multicolumn{5}{|c|}{20,000 generated scenarios } \\
\hline & 1 & 2 & 3 & 4 & 5 & 1 & 2 & 3 & 4 & 5 \\
\hline Objective func. & 0.00368 & 0.00354 & 0.00358 & 0.00346 & 0.00354 & 0.00352 & 0.00357 & 0.00352 & 0.00347 & 0.00352 \\
\hline $\begin{array}{l}\text { Investment in } \\
\text { FTSE (in \%) }\end{array}$ & 29.38 & 32.67 & 32.60 & 31.57 & 30.00 & 31.84 & 30.55 & 31.87 & 32.04 & 30.05 \\
\hline Gold (in \%) & 20.62 & 17.33 & 17.40 & 18.43 & 20.00 & 18.16 & 19.45 & 18.13 & 17.96 & 19.95 \\
\hline Bond (in \%) & 50.00 & 50.00 & 50.00 & 50.00 & 50.00 & 50.00 & 50.00 & 50.00 & 50.00 & 50.00 \\
\hline
\end{tabular}

Table 7 Investment problem with weight constraints, results for 10 scenario sets

\section{Backtesting}

To backtest the performance of our scenario generator and compare it to a standard GBM setting, we perform a rolling window backtest procedure to compare the out-of-sample performance. For this, we use the historical datasets of gold spots and the FTSE 100 index to generate scenario sets for 50 time steps. Each scenario set consists of 5,000 scenarios. We generate scenario sets for gold spots and FTSE 100 from the regime-switching model with a 3-state Markov chain. Furthermore we generate scenario sets for both time series from the model with a 1-state Markov chain, which represents a discretised version of the geomatric Brownian motion. For each of these 50 time steps, the optimisation is performed on the scenario sets from the regime-switching model as well as from the 1-state model, which does not allow switches between regimes. The optimal weights, which are obtained in these 100 optimisations (we obtain 50 sets of optimal weights for each model), are then utilised to create portfolios from the actual market data at that point in time.

The resulting portfolio values are plotted in figure 6 . We see here, that the resulting out-of-sample portfolio values in these 50 time steps are similar in both models. Nevertheless, a statistical analysis of both sets of portfolio values shows an outperformance of the regime-switching model with 3 possible states. The mean of the portfolio return from the backtest procedure in a 3-state Markov chain framework is higher than that obtained through GBM scenarios, the distribution of portfolio returns over that time period is more symmetric and it contains less outliers. This can be seen in the boxplots in figure 7 , the descriptive statistics are listed in table 8.

We furthermore perform an out-of sample stability test for the scenario generator. Large scenario sets are generated with a standard geometric Brownian motion method. These large scenario sets represent the "true" distribution of the gold and FTSE 100 returns. We fix the weights and $\alpha$ which we obtain from the optimal solution of one scenario set generated with our HMM scenario generator containing 5000 scenario sets. The fixed weights are 


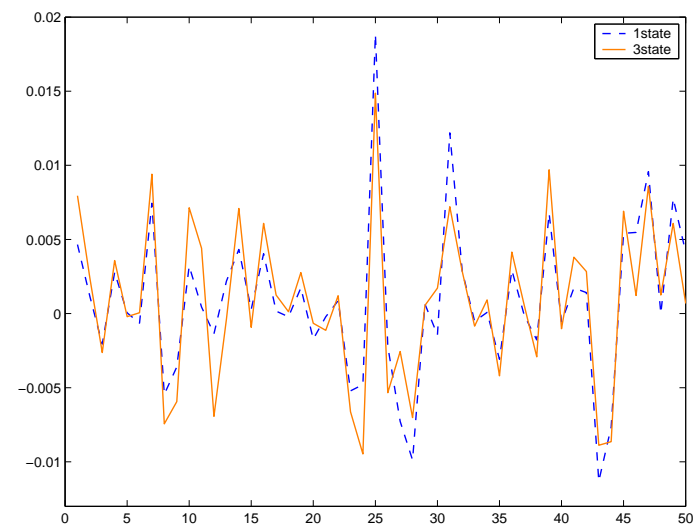

Fig. 6 Portfolio returns from rolling window backtest procedure

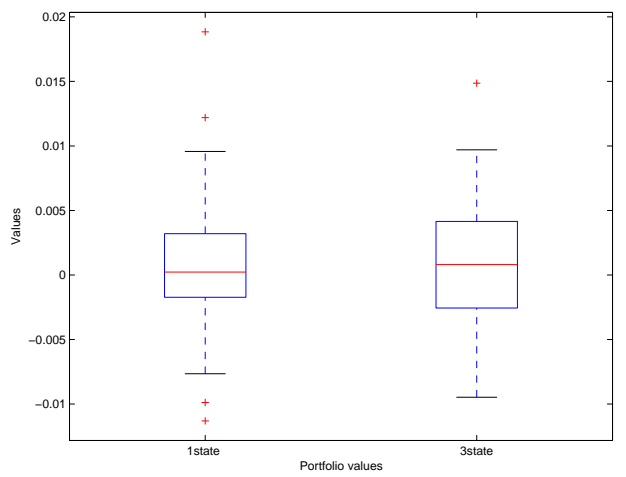

Fig. 7 Boxplots of both out-of-sample portfolio return series

\begin{tabular}{l|l|l|l|l|l|l|l}
\hline & Min & Max & Median & Mean & Standdev & Skew & Kur \\
\hline Portfolio returns 1-state & -0.0113 & 0.0188 & 0.0002 & 0.0008 & 0.0053 & 0.5748 & 4.9389 \\
Portfolio returns 3-state & -0.0095 & 0.0149 & 0.0008 & 0.0009 & 0.0054 & 0.0715 & 2.7587 \\
\hline
\end{tabular}

Table 8 Descriptive statistics of portfolio return series

\begin{tabular}{c|c}
\hline \hline FTSE & $8.81 \%$ \\
Gold & $22.37 \%$ \\
Bond & $68.82 \%$ \\
\hline \hline
\end{tabular}

and the resulting value of the objective function is 0.002898 . To test the out-of-sample stability, we perform the optimisation on scenario sets obtained from the GBM method with 30,000, 40,000 and 50,000 scenario sets respectively. The resulting objective functions are

\begin{tabular}{c|c} 
no. of scenarios & objective function \\
\hline \hline 30,000 & 0.1154564 \\
40,000 & 0.0025078 \\
50,000 & 0.00251607
\end{tabular}

The objective function obtained through the GBM scenarios becomes significantly smaller by increasing the number of scenarios, which is in accordance with the assumption that the "true" distribution is approached by the exogenous scenario generation method. The objective function obtained through the HMM scenario set is very similar to that obtained through the "true" distribution represented by 40,000 and 50,000 GBM scenarios.

We also perform an out-of-sample stability test for the optimal solution obtained in the problem setting with weight constraints. Here, the fixed weights from the HMM scenario setting are set to 


\begin{tabular}{c|c}
\hline \hline FTSE: & $31.57 \%$ \\
Gold: & $18.43 \%$ \\
Bond: & $50 \%$ \\
\hline \hline
\end{tabular}

with an objective function value of 0.00345911 . The out-of-sample test gives the following values for the objective function

\begin{tabular}{c|c} 
no. of scenarios & objective function \\
\hline \hline 30,000 & 0.05206740 \\
40,000 & 0.00295804 \\
50,000 & 0.00295804
\end{tabular}

Again, the objective function obtained through the HMM scenarios is very similar to the values obtained with 40,000 and 50,000 GBM scenarios. Therefore the HMM scenario generator seems to capture the "true" distribution of the gold and FTSE 100 returns.

\section{Conclusions}

In this paper, we modeled financial time series using a hidden Markov model approach. This enables the parameters of the observation process (which we assumed to be a discretised Geometric Brownian motion) to switch between different economic regimes, that are not directly observable. Thus our method, while not departing fundamentally from traditional approaches, tries to address a major shortcoming (the assumption that the parameters of the GBM are constant over time).

The parameters of the model are estimated using a filtering approach. Once the parameter estimation and the estimation of the Markov chain for the next time period are done, the model can be used for generating scenarios of future realisations for financial time series.

We tested the quality of the scenario generator in a financial decision making problem: a single period portfolio optimisation problem, in which the basket of assets is formed by FTSE 100, gold spots and a bank account. Scenarios are generated for the future returns of FTSE 100 and of gold spots under two frameworks: once, assuming that these time series are independent and follow different underlying Markov chains; secondly, assuming that the time series are correlated and governed by the same Markov chain. The scenarios generated were used as an input in a mean-CVaR optimisation problem. We tested the stability of the scenario generator by producing several different sets of scenario of the same size and solving the corresponding mean-CVaR model with each of these sets. We concluded that the scenario generator based on independent observations gives a good stability, both in-sample and, more importantly, out-of-sample. A comparison of scenario sets generated from the hidden markov model approach and scenario sets from a GBM setting is performed through a rolling window backtest. The portfolio returns obtained through the regime-switching model are in the mean higher than those from the GBM setting, the distribution of the HMM-based returns are less skewed.

The focus of future research is to extend and test the scenario generator for multiple time step optimisation problems.

Acknowledgements This work was carried out while Christina Erlwein was a NET ACE sponsored Intern at OptiRisk Systems; and Diana Roman was a KTP associate of Brunel Unversity assigned to OptiRisk systems. The support of these organisations are gratefully acknowledged.

\section{References}

Acerbi C, Tasche D (2002) On the coherence of Expected Shortfall. Journal of Banking and Finance 26:1487-1503

Akaike H (1974) A new look at the statistical model identification. IEEE Transactions on Automatic Control AC 19:716-723

Artzner P, Delbaen F, Eber J, Heath D (1999) Coherent measures of risk. Mathematical Finance 9(3):203228 
Bollerslev T (1986) Generalized Autoregressive Conditional Heteroskedasticity. Journal of Econometrics $31(2): 307-327$

Elliott R (1994) Exact adaptive filters for Markov chains in Gaussian noise. Automatica 30:1399-1408

Elliott R, Aggoun L, Moore J (1995) Hidden Markov models: estimation and control. Springer

Ellison E, Hajian M, Levkovitz R, Maros I, Mitra G (1999) A Fortran based mathematical programming system FortMP. Brunel University, Uxbridge, UK, NAG Ltd, Oxford UK

Engle RF (1982) Autoregressive Conditional Heteroskedasticity with Estimates of the Variance of U.K. Inflation. Econometrica 50(2):987-1007

Erlwein MR C, Davison M (2009) An examination of HMM-based investment strategies for asset allocation. Applied Stochastic Models in Business and Industry p doi: 10.1002/asmb.820

Fourer R, Gay DM, Kernighan B (1989) AMPL: A Mathematical Programming Language. AT\&T Bell Laboratories

Geyer A, Ziemba W (2007) The innovest austrian pension fund financial planning model innoalm. To appear in Operations Research, available at: http://edoc.huberlin.de/docviews/abstract.php?lang=ger\&id=26731

Hamilton JD, Susmel R (1994) Autoregressive Conditional Heteroskedasticity and Changes in Regime. Journal of Econometrics 64(2):307-333

Hardy M (2001) A Regime-Switching Model of Long-Term Stock Returns. North American Actuarial Journal 5(2):41-53

Hoyland K, Kaut M, Stein W (2003) A Heuristic for Moment-Matching Scenario Generation. Computational Optimization and Applications 24(2-3):169 - 185

Kaut M, Stein W (2003) Evaluation of scenario-generation methods for stochastic programming. Stochastic Programming E-Print Series (SPEPS) 14

Krokhmal P, Palmquist J, Uryasev S (2002) Portfolio optimization with conditional value-at-risk objective and constraints. The Journal of Risk 4(2):21-41

Mamon R, Erlwein C, Gopaluni R (2008) Adaptive signal processing of asset price dynamics with predictability analysis. Information Sciences 178(1):203-219

Markowitz H (1952) Portfolio selection. The Journal of Finance 7(1):77-91

Messina E, Toscani D (2007) Hidden markov models for scenario generation. IMA Journal of Management Maths Advance Access published online on October 8, 2007

Mulvey J (2001) Introduction to financial optimization: mathematical programming special issue. Mathematical Programming 89(2):205-216

Pflug G (2000) Some remarks on the value-at-risk and the conditional value-at-risk, probabilistic constrained optimization: methodology and applications. Kluwer Academic Publishers 38:272-281

Rabiner LR (1989) A tutorial on Hidden Markov Models and selected applications in speech recognition. Proc of the IEEE 77(2):257-286

Rabiner LR (2002) Hidden Markov Processes. IEEE Transactions on Information Theory 48(6):1518- 1569

Rockafellar R, Uryasev S (2000) Optimization of Conditional Value-at-Risk. Journal of Risk 2(3):21-41

Rockafellar R, Uryasev S (2002) Conditional value-at-risk for general loss distributions. Journal of Banking and Finance 26:1443-1471

Roman D, Mitra G, Spagnolo N (2008) Hidden markov models for financial optimisation problems. CARISMA Technical report CTR/77/08, available on-line at www.carisma.brunel.ac.uk, to appear in: IMA Journal of Management Maths

Ross S (2002) An Elementary Introduction to Mathematical Finance. Cambridge University Press

Zhang Y (2004) Prediction of financial time series with hidden markov models. M.Sc. Thesis, Simon Fraser University, China 